\title{
Formación integral en la cantera del Real Murcia C.F.: Tutorías psicológicas
}

\author{
Verónica Gómez-Espejo', Beatriz Aroca ${ }^{2}$, Francisco J. Robles-Palazón ${ }^{3}$ y Aurelio Olmedilla ${ }^{4}$ \\ ${ }^{1}$ Real Murcia C.F. Universidad de Murcia, España, ${ }^{2}$ Universidad de Murcia, España, ${ }^{3}$ CAFD. Universidad de Murcia, España, ${ }^{4}$ Real \\ Murcia C.F. Universidad de Murcia, España
}

RESUMEN: La intervención psicológica en deportes de equipo exige, para su óptima realización, un trabajo interdisciplinar (directivos, entrenadores, preparadores físicos, psicólogos/as, servicios médicos, padres y madres) que permita cubrir todas las necesidades de los deportistas. En el trabajo que nos ocupa, se muestra la importancia del asesoramiento y consultoría psicológica con jugadores de fútbol y entrenadores de las categorías inferiores del Real Murcia C. F. El entrenamiento individual y la orientación marcan la parte fundamental de la intervención psicológica desarrollada. El trabajo con los jugadores y entrenadores tiene como objetivo dotar a estos de una formación psicológica que les permita aprender estrategias y técnicas para aplicar en su práctica deportiva habitual, centrándose la intervención psicológica, principalmente, en el entrenamiento psicológico y la formación-educación. Se concluye que los programas de entrenamiento psicológico aplicados de manera individual con los futbolistas base dan resultados positivos, dotando a estos de técnicas y estrategias psicológicas para su correcto desempeño deportivo.

PALABRAS CLAVES: Intervención psicológica, trabajo interdisciplinar, fútbol base.

\section{Personal development in the Real Murcia C. F. Football academy: psychological mentoring}

ABSTRACT: Optimal psychological intervention in team sports requires an interdisciplinary work (managers, coaches, physical trainers, psychologists, medical services, parents) that allows for covering all the needs of athletes. This study shows the importance of psychological advice to coaches and young football players in the younger Real Murcia C.F. football players. Individual training and orientation represent the main part of the psychological intervention applied. Work with players and coaches is aimed at providing them with a psychological development that allows them for learning strategies and techniques to be used in their routine sports practice. Psychological intervention is mainly focused on psychological training and education/development. It is concluded that psychological training programs individually applied to young football players have positive results, providing them with adequate psychological techniques and strategies for sports performance.

KEYWORDS: Psychological intervention, interdisciplinary work, youth football.

\section{Formação integral no clube do Real Murcia C.F.: Tutoriais psicológicos}

RESUMO: A intervenção psicológica nos esportes em equipe requer, para seu ótimo desempenho, um trabalho interdisciplinar (dirigentes, treinadores, preparadores físicos, psicólogos, serviços médicos, pais) para atender a todas as necessidades dos atletas. Neste trabalho, é demonstrada a importância do aconselhamento e aconselhamento psicológico aos jogadores de futebol e treinadores das categorias mais baixas do Real Murcia C. F. O treinamento individual e a orientação marcam a parte fundamental da intervenção psicológica desenvolvida. O objetivo do trabalho com os jogadores e treinadores é capacitá-los com um treinamento psicológico que lhes permita aprender estratégias e técnicas para se aplicar na prática esportiva habitual, com foco na intervenção psicológica, principalmente no treinamento psicológico e no treinamento educacional. Conclui-se que os programas de treinamento psicológico

\footnotetext{
Verónica Gómez-Espejo es Psicóloga en el Real Murcia C.F. Universidad de Murcia; Beatriz Aroca es Psicóloga en la Universidad de Murcia; Francisco J. Robles-Palazón es CAFD en la Universidad de Murcia; Aurelio Olmedilla es Psicólogo en el Real Murcia C.F. Universidad de Murcia.

La correspondencia sobre este artículo debe enviarse a Verónica Gómez-Espejo. Universidad de Murcia. Campus de Espinardo. 30100 Espinardo, Murcia. Email:
}

(cc) EY-NC-ND Este es un artículo Open Access bajo la licencia 
aplicados individualmente aos jogadores juvenis dão resultados positivos, equipando-os com técnicas psicológicas e estratégias para seu desempenho esportivo correto.

PALAVRAS-CHAVE: Intervenção psicológica, trabalho interdisciplinar, futebol juvenil.

Artículo recibido: 11/09/2017 | Artículo aceptado: 18/11/2017

La importancia de la intervención psicológica en el deporte hoy día es un hecho, ya no solo para la optimización del rendimiento deportivo o un mayor bienestar psicológico (Lameiras, Almeida, Pons y García-Mas, 2014; Latinjak, Font-Lladó, Zourbanos y Hatzigeorgiadis, 2016; Olmedilla, Bazaco, Ortega y Boladeras, 2011; Salgado, Rivas y García-Mas, 2011; Sheard y Golby, 2006), sino por la enseñanza de técnicas y estrategias psicológicas que llevan a los deportistas a mejorar sus habilidades psicológicas básicas necesarias para la práctica deportiva como la concentración, la autoconfianza, el control del estrés, o la motivación entre otras muchas (Cruz, 1992; Cruz, 1994; Junichi y Hajime, 2007; Olmedilla y Domínguez-Igual, 2016).

El trabajo psicológico, generalmente basado en la terapia cognitivo-conductual (McArdle y Moore, 2012; Turner, Slater y Barker, 2014; Weinberg y Gould, 2010) ha sido poco a poco insertado en el plan de entrenamientos de equipos y deportistas (Chicau, Silva y Palmi, 2012; García-Naveira y Jerez, 2012). Diferentes publicaciones muestran la eficacia de programas de intervención psicológica para el control y estabilidad emocional (Olmedilla, Ortega, Andreu y Ortín, 2010), para el aprendizaje de técnicas y estrategias que aumenten las habilidades psicológicas de los deportistas (Buceta, 2007; Lorenzo, Gómez, Pujals y Lorenzo, 2012), o para poner de manifiesto la satisfacción de los deportistas con la intervención psicológica (Olmedilla, Ortega, Boladeras, Ortín y Bazaco, 2013), así como la eficacia de las técnicas de control de la activación, relajación y visualización (Jordet, 2005; Munroe-Chandler, Murphy, Hall y Fishburne, 2007; Olmedilla et al., 2010; Wood y Wilson, 2012; Wright, McCormick, Birks, Loporto y Holmes, 2015).

Por otro lado, tal y como indican Abenza et al. (2014) para llevar a cabo una intervención psicológica es primordial conocer el contexto en el que se va a realizar la preparación psicológica así como las características y peculiaridades de la modalidad deportiva donde se va a intervenir. En la misma línea, Olmedilla et al. (2011) señalan que "el psicólogo cuando trabaja con deportistas de forma individual pueda tener un feedback más directo y fiable, que le permite observar la mejoría, en su caso, de diferentes aspectos psicológicos trabajados, así como la satisfacción del deportista con el trabajo y la aplicabilidad de lo aprendido en diferentes contex- tos... sin embargo, cuando el psicólogo trabaja con grupos o equipos deportivos puede no tener una visión tan clara del proceso de entrenamiento psicológico, apareciendo otras variables (relaciones entre los miembros, cooperación y cohesión, rendimiento individual y de equipo, resultados, etc.) que dificultan dicha percepción" (p. 222).

Cabe resaltar cómo la inclusión del entrenamiento psicológico cada día se hace más patente dentro del entrenamiento deportivo, ya que esto no era habitual en la mayoría de países, incluido el nuestro, no muchas décadas atrás. Como menciona Cruz (1992) "la Psicología del Deporte ha evolucionado de un primer periodo en el que predominaban los estudios de laboratorio sobre aprendizaje motor y los estudios descriptivos acerca de la personalidad del deportista a un segundo periodo de aplicaciones prácticas en el que se concede la máxima importancia al entrenamiento psicológico de los atletas para aumentar el rendimiento deportivo" (p. 17).

Además, actualmente se observa cómo el entrenamiento psicológico cada vez se realiza en un mayor número de deportes, tanto a nivel individual como en equipos deportivos, y no solo en deporte profesional, sino también en la tecnificación deportiva de jóvenes promesas y en el deporte de base (Lorenzo et al., 2012; Pujals y Jodra, 2011; Romero et al., 2010).

Asimismo, una de las áreas de desarrollo de la Psicología del Deporte aplicada que más repercusión está adquiriendo hoy día es el fútbol base, no solo como medio de mejora de la salud y de las capacidades motoras de los practicantes, sino por la importancia que tiene en el proceso de socialización de los jóvenes (Hernández, 2005). El entorno deportivo se convierte en un medio de influencias socializadoras que juegan un papel destacado en la formación del niño (Torregrosa y Cruz, 2006). Aquí se puede hacer mención del llamado "triángulo deportivo", que está formado por el deportista, el entrenador y la familia, aunque no solo ellos influyen en la participación y la calidad de la experiencia deportiva de los jóvenes deportistas, también ha de considerarse el grupo de iguales así como los árbitros o los organizadores de las competiciones deportivas (Boixadós y Valiente 2007). Por todo ello, los riesgos y/o beneficios de la participación deportiva dependen de las exigencias y las expectativas de 
los entrenadores y los padres, así como de los objetivos que se propongan los organizadores de las competiciones deportivas (Cruz 1994). Tal y como indican diferentes investigaciones (Andreu, 2003; Vives y Garcés, 2003), los efectos positivos que se originan de la práctica de un deporte como el fútbol en edades tempranas dependen de cómo se oriente la actividad deportiva, de la importancia que se otorgue al aprendizaje y al entrenamiento psicológico, ya que no surgen de un modo espontáneo. Es necesario que se estructuren los medios necesarios para coordinar todas las acciones que fomenten rendimiento, aprendizaje, bienestar, y desarroIlo personal; solo de ese modo se accederán a importantes beneficios a través de la práctica deportiva, siendo éste el objetivo que se pretende mediar a través de la actuación psicológica en un equipo de fútbol base. Es aquí donde la Psicología del Deporte se presenta como un recurso más.

Partiendo desde esta perspectiva y aprendizaje, a continuación se expone la historia del área de Psicología del Real Murcia Club de Fútbol, así como los diferentes servicios prestados por esta, el protocolo de actuación de los usuarios, las problemáticas más frecuentes y varios ejemplos de casos. Todo ello con la finalidad de que pueda servir de referente para futuros trabajos o la mejora de la profesión.

El presente artículo es un resumen del trabajo de intervención psicológica llevado a cabo en el Real Murcia Club de Fútbol a lo largo de la temporada 2016-2017, y la estructura básica de la actual temporada (2017-2018). Se pretende dar a conocer brevemente la labor realizada con el fin de contribuir a un mayor reconocimiento de la envergadura que posee la intervención o asesoramiento psicológico en el ámbito deportivo.

\section{Área de Psicología del Real Murcia C.F.}

El Área de Psicología del Real Murcia C.F. surgió de las diferentes acciones a desarrolladas gracias al convenio de colaboración firmado en el año 2014 entre la Factoría Grana (anteriormente Fundación Real Murcia) y la Universidad de Murcia (UMU). Ésta pretende ser una herramienta facilitada por el club para ayudar en la formación integral de sus jóvenes futbolistas, y por ende, para obtener una mejora en el rendimiento deportivo de los mismos, a través de la formación de los propios jugadores y de los diferentes agentes que median con ellos (entrenadores, padres-madres y el propio club). Por tanto, y para conseguir estos objetivos, se firmó un contrato entre la Factoría Grana y el Departamento de Personalidad, Evaluación y Tratamiento Psicológico de la Universidad de Murcia para el desarrollo del presente programa.

\section{Ámbitos de Actuación}

La estructura y acciones están centradas en las categorías de Fútbol Base del club, desde la categoría de debutantes (4-5 años) hasta el filial del Real Murcia (3ª división), donde el trabajo se realiza con los jugadores, los entrenadores y los padres y madres de éstos. Aunque a priori pudiera parecer que el rango de edad es bastante amplio, los principios básicos que imperan en nuestra intervención son los mismos, adaptados en función de la situación (competición, entrenamiento...), de las diferencias individuales de cada uno de los usuarios (edad, sexo, categoría deportiva...) y de sus requerimientos (rendimiento deportivo y académico, educación, formación...).

Las competencias del Área de Psicología en el Real Murcia C.F., sus funciones y los profesionales que a ello se dedican, se han ido ampliando y extendiendo en función de los requerimientos y necesidades que desde el club y por los propios usuarios y directivos se encontraban. De este modo y con el trabajo de 5 psicólogos que en ella participan, para las temporadas 2016-2017 y 2017/2018, el plan de trabajo diseñado ha sido el siguiente:

a) Escuela de padres

La influencia que ejercen los padres y otros significativos en el proceso deportivo de sus hijos es notable. Su comportamiento se asocia con la elección de la modalidad deportiva, la orientación motivacional e incluso los resultados deportivos de sus hijos. En este contexto es fundamental que los padres tengan presente cuáles son sus funciones más importantes en la iniciación y práctica deportiva de sus hijos. Lo que se pretende es proporcionar a los padres/madres un espacio de reflexión y debate que les permita sensibilizarse del papel que desempeñan en la práctica educativo/ deportiva de sus hijos, para que puedan contribuir de forma positiva en la formación integral de éstos y que sus acciones se constituyan dentro del marco de actuaciones establecido por el club. Se podría decir que la mejor intervención es la prevención, es decir, evitar que se produzcan comportamientos inadecuados (Peris, 2008). Para una revisión sobre la temática ver los trabajos de Cruz (2001), Cruz, Figueroa, Garcia-Mas y Torregrosa (2003), Gimeno (2007) o Buceta (2015). En este sentido, las acciones son las siguientes:

- Sesiones informativas: se llevan a cabo en momentos puntuales de la temporada y se establecen las normas de funcionamiento del club y del equipo, protocolos de actuación, etc.

- Sesiones formativas: se llevan a cabo a lo largo de la temporada tres sesiones de psicoeducación para padres con temas como: qué es el entrenamiento psicológico, beneficios del deporte para mi hijo, papel de los padres en el 
deporte, cómo fomentar la motivación de mis hijos en la práctica deportiva, la comunicación entre padres e hijos, cómo integrar el deporte en la rutina diaria de mis hijos (estudios), favorecer la autonomía y responsabilidad de mis hijos.

b) Psicoeducación para entrenadores y demás miembros del cuerpo técnico

Son múltiples los trabajos que ponen de relieve la importancia de entrenar mentalmente a los entrenadores (García-Naveira, 2017; Ortín, Maestre y García-de-Alcaraz, 2016; Ramírez y Díaz, 2004; Sousa, Cruz, Torregrosa, Vilches y Viladrich, 2006; Sousa, Cruz, Viladrich y Torregrosa, 2007), ya que, por ejemplo, son estos los que asumen el reto de guiar a un grupo de niños en sus primeras etapas de acercamiento a la actividad física y al deporte, y por tanto, han de afrontar dicha tarea con una enorme responsabilidad educativa/formativa. Cualquier gesto, expresión, consejo u opinión que se lance a este pequeño y curioso público será absorbido de inmediato por sus mentes inquietas. Es por esto que una de las primeras misiones del entrenador debería centrarse en la adquisición de los conocimientos psicológicos necesarios para poder conducir acertadamente el aprendizaje de los niños que se encuentren bajo su tutela.

Lo que se pretende es que su desempeño trascienda de lo puramente deportivo y lleguen a la persona, abarcando diferentes áreas del jugador (García-Naveira y Jerez, 2012). En este sentido, las acciones son las siguientes:

- Sesiones formativas: se llevan a cabo a lo largo de la temporada nueve sesiones (mensualmente) de psicoeducación con entrenadores en las que se tratan cuestiones como: la motivación (propia y de sus jugadores), activación, concentración, refuerzos para el aprendizaje/rendimiento, sentimiento de club, dinámicas de equipo, relación entre técnicos y padres, etc.

- Reuniones periódicas: con el fin de intercambiar información y opiniones sobre el funcionamiento individual y colectivo de los jugadores y del propio entrenador, así como poner en su conocimiento el reglamento del club, protocolos de actuación, etc.

c) Programa de entrenamiento psicológico orientado a los equipos de rendimiento.

El programa de entrenamiento psicológico pretende ayudar al futbolista en su formación integral, en sus momentos de desconcierto o desmotivación propios en el desarrollo de su vida deportiva, intentando evitar su abandono y ayudando a asentar políticas de formación de talentos para nutrir los equipos de categoría superior. Para una revisión sobre la temática destacar los trabajos de autores como Buceta (1992), Cruz (1992) o Bossio, Raimundi y Correa (2012).

Desde el Área de Psicología se lleva a cabo un programa de entrenamiento psicológico específico con las categorías juvenil y cadete del club (a excepción del Juvenil División de Honor, con el que se hace un trabajo más específico, detallado a continuación). El principal objetivo del programa de entrenamiento psicológico es mejorar las aptitudes psicológicas del deportista para la competición. Para ello se lleva a cabo lo siguiente:

- Programa de entrenamiento psicológico. Compuesto por un total de 9 sesiones ( 1 al mes) de 60 minutos con cada uno de los equipos en las que se tratan aspectos como: conocer a los jugadores y evaluar sus aptitudes psicológicas, técnicas para el establecimiento de objetivos, relajación, visualización y activación. Para facilitar la transferencia de conocimientos al terreno de juego, algunas de las sesiones son implementadas en el campo de futbol (p. ej., regular el nivel de activación en diferentes momentos del juego).

- Psicología para la salud aplicada al deporte. Además de la parcela deportiva, se abordan también problemas y aspectos personales del deportista; por tanto, en caso de que el psicólogo considere necesario una intervención psicológica más específica en el deportista, éste será derivado a un trabajo individual, denominado "tutorías psicológicas individuales".

d) Trabajo psicológico específico con el Juvenil División de Honor.

Lo que se pretende con esta intervención es abordar y estudiar aquellas facetas deportivas y personales que inciden tanto en la actividad familiar y social, como en la puramente futbolística, teniendo en cuenta estudios de diversos autores que anteriormente han trabajado con deportistas (Godoy-Izquierdo, Vélez y Pradas 2009; Lorenzo et al., 2012; Olmedilla, Ortega, Ortín y Andreu, 2008). Así, una psicóloga de manera semanal y continua acude a los entrenamientos y competiciones para analizar en los futbolistas las relaciones personales con la familia, el tiempo de dedicación al estudio y el seguimiento académico, sin olvidar aquellos aspectos psicológicos que repercuten claramente a su actividad deportiva, como son la motivación, atención-concentración, el nivel de ansiedad antes, durante y después de la competición y las posibles dificultades asociados a ellos. En definitiva, lo que se pretende es aportar al entrenador y demás cuerpo técnico una serie de datos sobre distintas facetas psicológicas del joven futbolista para que, en caso de considerarlo necesario, se pueda intervenir de manera óptima con el jugador.

\section{e) Tutorías psicológicas individuales.}

Son llevadas a cabo por la psicóloga del club y están destinadas a entrenadores y demás miembros del cuerpo técnico, jugadores y padres/madres de los mismos, que pueden venir por iniciativa propia o derivados por otros. Dichos servicios son prestados una tarde a la semana y tiene una duración de 45 minutos. El objetivo de estas sesiones individua- 
les es solucionar los posibles problemas que presenten los usuarios y que, consecuentemente, pueden influir, de manera directa o indirecta, en el correcto desarrollo de la práctica deportiva de los jugadores. Para conocer otros trabajos que muestran la utilidad de estos servicios podemos remitirnos a Barker, Mellalieu, McCarthy, Jones y Moran (2013) y Olmedilla y Domínguez-Igual (2016).

De este modo, el psicólogo cuando trabaja con deportistas de forma individual puede tener un feedback más directo y fiable que le permite observar la mejoría, en su caso, de diferentes aspectos psicológicos trabajados, además de la satisfacción del deportista con el trabajo y la aplicabilidad de lo aprendido en diferentes contextos.

f) Observación y registro de sesiones de entrenamiento y competición.

Como afirma Bakeman y Gottman (1989) el estudio de ciertos problemas, tal como es el caso del deporte, requieren una perspectiva dinámica de la conducta social en lugar de una estática. Por ello, semanalmente, una de las psicólogas asiste a los campos de entrenamiento situados en diferentes pueblos y pedanías de Murcia con el objetivo de observar las sesiones de entrenamiento en búsqueda de posibles necesidades que pudieran surgir y no hubieran sido detectadas por los entrenadores y el resto del cuerpo técnico. Se actúa con aquellos entrenadores que ya han detectado alguna necesidad en sus jugadores o crean necesaria su actuación de manera tanto individual como grupal, realizando el seguimiento de la evolución de los equipos y de los casos individuales tratados, por medio del contacto directo con los entrenadores y la observación continua de las sesiones de entrenamiento. El objetivo es cubrir cualquier posible necesidad que hubiera sido detectada con anterioridad, así como la detección de aquellas necesidades que no hayan sido reflejadas por su complejidad. Otros autores también destacan la importancia de esta labor, para ello podemos atender a trabajos como los de Morilla et al. (2001), Medina y Delgado (1999) o Blasco (1994).

g) Investigación/divulgación

Todas las acciones son susceptibles de iniciar líneas de investigación específicas, que repercutirán positivamente en el desarrollo de las acciones para próximas temporadas. Se implementarán tanto las propuestas desde el Área de Psicología, como las que vengan del club y sean viables. Dicho trabajo ha sido y será expuesto a través de diferentes medios (Congresos, Jornadas, Publicaciones, etc.) para que pueda ser de utilidad a otros profesionales interesados. Dosil (2004), ya en el primer capítulo de su libro "Psicología de la Actividad Física y del Deporte", refiere que la reflexión y la investigación son absolutamente indispensables para entender la realidad de la que se ocupa una ciencia. Otros au- tores como Groves, Heekin y Banks (1978) o Lorenzo (2001), también pusieron de relieve la importancia de la investigación en el terreno de la Psicología del Deporte en aras de un mayor y mejor avance de la misma.

En la Figura 1 se puede observar los diferentes ámbitos de actuación del Área de Psicología del Deporte del club.

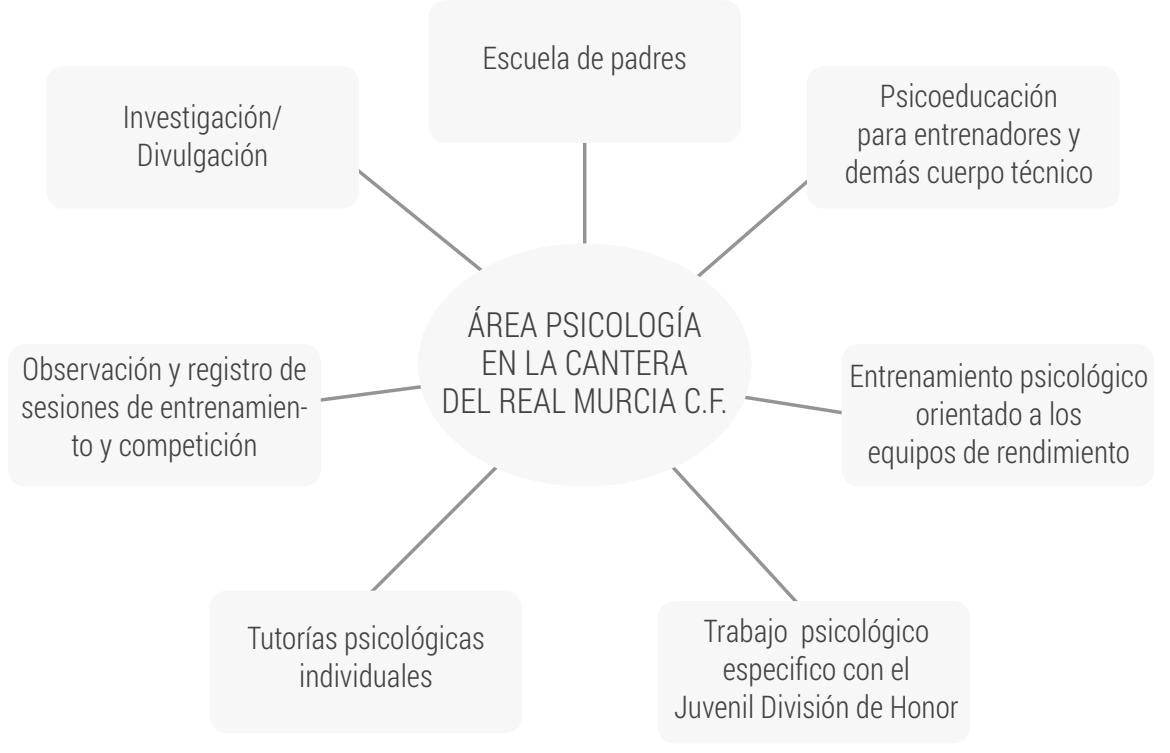

Figura 1. Ámbitos de actuación del Área de Psicología del Deporte del Real Murcia C. F.

\section{Recursos Humanos y Físicos Disponibles}

La estructura del Área de Psicología del club es la siguiente: - 1 Psicólogo, Director del Área de Psicología. Supervisa y dirige todas las acciones del área. Imparte las sesiones formativas con todos los entrenadores del fútbol base.

- 1 Psicóloga, Coordinadora del Área de Psicología. Realiza las tutorías psicológicas individuales, el trabajo psicológico específico con el juvenil División de Honor y la observación y registro de las sesiones de entrenamiento y competición.

- 2 Psicólogos, encargados de las Sesiones formativas con padres.

- 1 Psicóloga, encargada del programa de entrenamiento psicológico orientado a los equipos de rendimiento.

El resto de funciones son desarrolladas por el conjunto del equipo, imperando la interdisciplinariedad entre los diferentes agentes interesados que participan en el club (directivos, coordinadores, servicio médico, área de metodología, etc.).

Para realizar estas acciones se cuenta actualmente con los siguientes medios físicos: un despacho, una sala polivalente, dos salas de reuniones y los dos campos de futbol (futbol 8 y 11) donde entrenan los equipos. 


\section{Tutorías Psicológicas Individuales}

En los últimos años cada vez con más frecuencia se habla del papel decisivo de los factores psicológicos en el resultado deportivo. $Y$ aunque las actividades tratadas hasta el momento hacen referencia principalmente a una intervención psicológica grupal, es necesario destacar la importancia del tratamiento psicológico individualizado a través de las tutorías psicológicas.

La preparación psicológica puede ser entendida como un proceso para incrementar el rendimiento deportivo mediante el desarrollo de un control efectivo sobre las variables de orden psicológico relacionadas con el logro futbolístico, formando además recursos y cualidades útiles para el desempeño del futbolista en otros ámbitos de su vida (Valdés, 1996). El entrenamiento psicológico se puede considerar como una forma más del entrenamiento deportivo que incide directamente en los procesos de formación de los deportistas (Olmedilla et al., 2011). Es una forma más del entrenamiento deportivo que, poco a poco, se ha ido incluyendo en el método de trabajo de muchos deportistas y equipos deportivos (Chicau et al., 2012; García-Naveira y Jerez, 2012; Jaenes, Caracuel y Penaloza, 2012). Teniendo, por tanto, el psicólogo que trabajar para que los deportistas mejoren el dominio de las habilidades psicológicas que requiere cada especialidad deportiva, permitiendo al deportista adquirir técnicas y estrategias psicológicas que faciliten los procesos de reflexión y toma de decisiones, teniendo en consideración tanto el ámbito deportivo como el extradeportivo

De este modo, el psicólogo cuando trabaja con deportistas de forma individual puede tener un feedback más directo y fiable que le permite observar la mejoría, en su caso, de diferentes aspectos psicológicos trabajados, además de la satisfacción del deportista con el trabajo y la aplicabilidad de lo aprendido en diferentes contextos (Barker et al., 2013; Olmedilla y Domínguez-Igual, 2016).

Es por esto que desde el Área de Psicología, con el apoyo del club, se establece como objetivo prioritario velar por el bienestar de los jugadores y potenciar sus capacidades deportivas, partiendo de la base de que el rendimiento deportivo es multifactorial. Cuando se atiende a un deportista, se busca atender las diferentes demandas que pueda tener, tanto en su vertiente personal (bienestar, problemas personales, familiares...) como deportiva (rendimiento, lesión...) (Garcia-Naveira, 2010).

Para una mayor comprensión del funcionamiento de este servicio a continuación se detallan algunos aspectos del mismo para finalizar con tres ejemplos prácticos reales que muestran el modo de intervención en estos casos concretos.

\section{a) Población diana:}

Todos los usuarios pertenecientes a la Factoría Grana ya sean jugadores, entrenadores y demás cuerpo técnico, padre/madres de los jugadores, directivos, etc., pueden solicitar la asistencia de la psicóloga a través de las tutorías psicológicas.

Actualmente el club cuenta con más de 30 equipos en sus bases, que van desde la categoría debutantes hasta $3^{a}$ división. Y son más de 60 las personas que conforman el staff técnico de estos diferentes equipos (entrenador, entrenador de porteros, $2^{\circ}$ entrenador, fisioterapeutas, preparador físico, delegado, etc.).

b) Protocolo de actuación

Como ya se ha indicado anteriormente, al inicio de temporada se da cita a los padres/madres y entrenadores (en actividades separadas) a una sesión formativa en la que se muestra el protocolo de actuación en caso de necesitar tutorías psicológicas individuales.

- Cuando son los técnicos quienes detectan el problema: 1) informar a los padres, hablándole de los servicios de Psicología del club e indicándoles la utilidad de recurrir a los mismos, 2) paralelamente comunicarse con el Área de Psicología para reportar este problema y 3 ) seguidamente serían los padres los que habrían de solicitar este servicio. - Cuando son los padres quienes detectan el problema: 1) informar al entrenador del comportamiento o conducta que les preocupan y 2) ponerse en contacto con el Área de Psicología.

- Cuando la petición la hace un jugador mayor de edad o un técnico, este debería ponerse en contacto directamente con el servicio de psicología.

En todo momento se mantiene el secreto profesional y la confidencialidad de las sesiones, salvo que por requerimientos de la intervención se necesite lo contrario, para lo cual se pedirá explícitamente permiso a los interesados y responsables.

\section{c) Medios físicos y humanos para su realización}

Estos servicios son prestados por una psicóloga que atiende de manera individual una tarde a la semana, en horario de oficina (16.30-19h) en un despacho situado en las oficinas del Estadio Nueva Condomina (Murcia). Los usuarios que acuden a las tutorías lo hacen con cita previa, solicitada a través de contacto directo con la psicóloga vía telefónica y su duración es de 45 minutos.

Al trabajar en la mayoría de las ocasiones con menores de edad y al tratarse en ocasiones de cuestiones de diversa índole, algunas que salen de los aspectos puramente deportivos, ha sido necesario contar con la colaboración de los entrenadores y/o padres, para el correcto desarrollo de las intervenciones. 


\section{d) Principales problemáticas tratadas}

Las dificultades principales que se tratan son: problemas de autoestima y autoconfianza, dificultades de comportamiento, falta de autonomía en la realización de las tareas en casa, dificultades de aprendizaje, problemas de motivación, dificultades en la gestión de la frustración, entre otras.

e) Ejemplos de casos prácticos:

El proceso de entrenamiento psicológico, al igual que todo entrenamiento, implica la consideración de al menos tres componentes (Ureña, 2003): 1) Determinar y valorar el perfil psicológico del deportista en relación con la práctica de un deporte específico, situación que implica la identificación de las condiciones y características actuales del deportista; 2) Identificar las destrezas psicológicas que requiere el deportista para lograr un rendimiento máximo en la práctica de un deporte específico, es decir, el estado al que se quiere llevar al deportista; y 3) Establecer la estrategia metodológica a seguir en la transición del estado actual del deportista hasta el que se le quiere llevar, al respecto es preciso empoderarse de los conocimientos y técnicas precisas para promover el desarrollo y consolidación de las destrezas psicológicas necesarias para la práctica exitosa de un deporte específico. De este modo, el entrenamiento de cualquier factor psicológico-deportivo pasaría por esos tres momentos.

\section{Caso 1: Problemas de Autoconfianza}

El programa de intervención se llevó a cabo la temporada 2016/2017 con un futbolista de 15 años (categoría cadete) que jugaba al futbol desde los 7 años. Pasó a formar parte de la cantera del Real Murcia en la categoría benjamín (9 años). Entrenaba 3 días semanales (1,5 horas cada entrenamiento), compaginando la práctica del futbol con sus estudios de secundaria. La demanda de atención fue realizada por el propio jugador a través de sus padres que se pusieron en contacto con el servicio de psicología.

La demanda del jugador fue mejorar su rendimiento deportivo, especialmente en las competiciones ya que "cuando salgo a jugar siento que voy a fallar, y cuando algo no me sale como yo quiero...me pierdo...y eso me hace fallar una y otra vez". De esta manera y tras una primera sesión en la que se administró el CPRD de Gimeno, Buceta y Pérez-Llantada (2001) y se llevó a cabo la entrevista inicial, se propuso un programa de entrenamiento psicológico centrado en la mejora de la autoconfianza, basado en el programa de entrenamiento usado por Olmedilla y Dominguez-Igual (2016). El programa duró 12 semanas en las que se llevaron a cabo un total de 10 sesiones de 1 hora de duración, y se basó en la Terapia Cognitiva Conductual (McArdle y Moore, 2012; Turner et al., 2014), la cual indica que las distorsiones y sesgos cognitivos influyen en gran parte de los problemas psicológicos.

Por tanto, la intervención estuvo formada por técnicas cognitivo-conductuales como la reestructuración cognitiva, auto-registros conductuales, establecimiento de objetivos y técnicas de relajación y visualización. De este modo, el plan de trabajo que se siguió fue el siguiente:

1 Evaluación psicológica inicial. Determinar el perfil psicológico y posibles vías de actuación.

2 Intervención psicológica. Mediante la fase educativa, el entrenamiento de habilidades y técnicas y la aplicación de las mismas.

3 Evaluación psicológica final. Determinar la eficacia del programa y la satisfacción del deportista respecto al mismo.

Los resultados fueron alentadores ya que, en general, se puede afirmar que el deportista mejoró su nivel de autoconfianza en la práctica deportiva, volviendo a la normalidad en los entrenamientos y competición tras la aplicación del programa de entrenamiento.

\section{Caso 2: Control de la Ansiedad}

El programa de intervención se está llevando a cabo la temporada 2017/2018 con un futbolista de 9 años (categoría benjamín) que juega al fútbol desde los 5 años. Esta temporada es su primera temporada en la Factoría Grana. Entrena 3 días semanales (1,5 horas cada entrenamiento), compaginando la práctica del futbol con sus estudios de primaria. La demanda de atención fue realizada por los padres del jugador, orientados por el entrenador.

Los padres referían que "en los entrenamientos lo hace de maravilla, pero cuando llega la competición....se agobia... se queda paralizado y no quiere jugar", el entrenador dice que "sale del partido con cara de angustiado, muy nervioso, parece que se le vaya a salir el corazón”. De esta manera y tras una primera sesión en la que mantuvimos una entrevista inicial con los padres y el entrenador, se decidió crear un programa de entrenamiento de 2 meses de duración (actualmente en proceso) en el que llevaremos a cabo un total de 8 sesiones de 1 hora de duración (aproximadamente). Este programa se basa en la Terapia Cognitiva Conductual, y se utiliza prioritariamente el uso de la relajación y las tareas para casa. De este modo, el plan de trabajo que se siguió fue el siguiente:

1 Evaluación psicológica inicial. Se evaluó el perfil psicológico inicial con el STAI-C (Spielberger, 1973) para niños que permitió, mediante los resultados, estructurar el programa de entrenamiento. 
2 Intervención psicológica. El objetivo es que el jugador aprenda a discriminar e identificar las respuestas generadas por la situación ansiógena y entrenarle para poner en marcha las habilidades de relajación, ayudándose de la imaginación y de las tareas para casa (Olivares, Xavier y Lozano, 2010).

3 Evaluación psicológica final. Determinar la eficacia del programa y la satisfacción del jugador hacia el mismo.

Al tratarse de un jugador de corta edad es preciso que los padres y entrenador muestren su participación y colaboración continua en el proceso de entrenamiento psicológico, algo que hasta el momento se está produciendo, sobre todo, en las tareas para casa.

Actualmente y aunque el programa todavía está en curso (mitad del proceso), podemos decir que, en líneas generales, se ha notado un cambio significativo en el comportamiento del futbolista respecto a la competición.

\section{Caso 3: Recuperación de Lesión}

El programa de intervención se llevó a cabo la temporada 2016/2017 con un futbolista de 20 años (3 ${ }^{a}$ división) que jugaba al fútbol desde los 5 años. Entrenaba 4 días semanales (1,5 horas cada entrenamiento), compaginando la práctica del fútbol con sus estudios universitarios. La demanda de atención fue realizada por el propio jugador.

Sufría una lesión grave en el hombro por dislocación y posterior cirugía reparadora, que se había enlazado con otra lesión anterior en la rodilla, lo que le mantenía alejado del juego 7 meses. Refería tener "miedo y ansiedad ante otra lesión que me pueda retirar definitivamente del fútbol", además mostraba necesidad de utilizar una férula de sujeción para el hombro, habiéndole sido indicado por el fisioterapeuta lo innecesario de usarla. "Necesito ponérmela, si no, no me siento cómodo, evito el choque y no juego como siempre".

Tras una primera sesión en la que se llevó a cabo una entrevista y una evaluación psicológica inicial con el CPRD
(Gimeno et al., 2001), y a tenor de los resultados, se decidió crear un programa de entrenamiento de 10 sesiones de 1 hora de duración (aproximadamente). Se aplicó un programa cognitivo-conductual cuyo propósito fue la reestructuración cognitiva, bajo el modelo de Beck (1976). De este modo, el plan de trabajo que se siguió fue el siguiente:

1 Evaluación psicológica inicial. Se evaluó el perfil psicológico inicial con el CPRD, que permitió, mediante los resultados, estructurar el programa de entrenamiento.

2 Intervención psicológica. Se llevó a cabo una Reestructuración Cognitiva, mediante el registro de pensamientos automáticos, cuya finalidad fue acabar con las distorsiones cognitivas (sobregeneralización y magnificación), la cual se hizo en tres fases (educativa, de entrenamiento y de aplicación). En la Tabla 1 se muestra una ejemplo real del autorregisto, en el cual, las dos últimas columnas se corresponden con la segunda fase de aplicación en la que el jugador ha de reformular los pensamientos distorsionados.

3 Evaluación psicológica final. Determinar la eficacia del programa y la satisfacción del jugador hacia el mismo.

Los resultados fueron positivos. El deportista mejoró su nivel de autoconfianza en el deporte respecto a la práctica del mismo sin la férula, volviendo a la normalidad en los entrenamientos y competición y reportando volver a la práctica deportiva tras la lesión al mismo nivel de rendimiento percibido tanto psicológico como físico.

\section{Conclusión}

El objetivo de este documento ha sido dar a conocer el trabajo psicológico que se realiza en las categorías inferiores del Real Murcia C.F. Se han mostrado concretamente algunas de las actuaciones específicas que mediante programas de entrenamiento psicológico se han llevado y se están llevando a cabo con los distintos deportistas para que aprendan técnicas y estrategias psicológicas, y las apliquen para mejorar sus propias habilidades.

Tabla 1. Ejemplo real de autorregistro. "Registro de pensamientos"

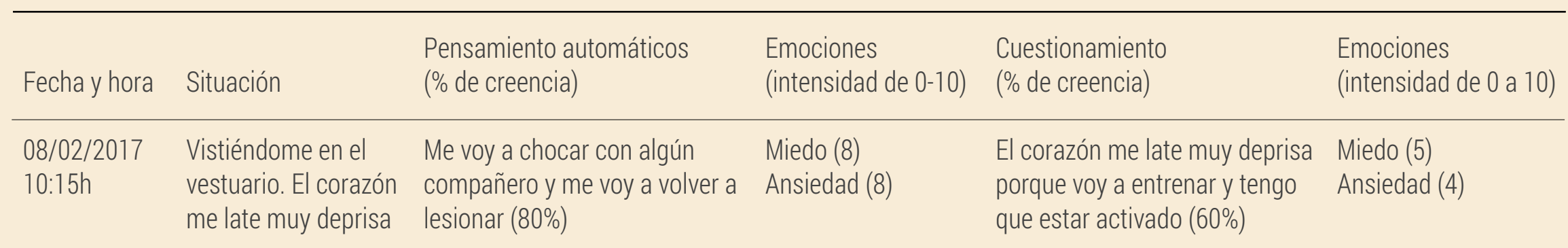


Los resultados de los programas de entrenamiento psicológico aplicados (y llevados a término) con los jugadores y mostrados en los ejemplos de casos prácticos han cumplido con los objetivos para los que fueron diseñados si comparamos los resultados de la evaluación psicológica inicial con la final, tanto en los entrenamientos como en la competición, logrando mejorar el manejo de las habilidades psicológicas relacionadas con el rendimiento deportivo. Además el nivel de satisfacción de los jugadores, padres y entrenadores respecto a los resultados de los programas de entrenamiento psicológico fue muy bueno.

Sin embargo, estos programas de entrenamiento se limitan al entrenamiento psicológico del deportista, sin tener en consideración la actuación necesaria y paralela en el tiempo con los entrenadores y padres. Del mismo modo resulta también evidente la necesidad de programar el entrenamiento de las habilidades psicológicas dentro el contexto deportivo, y de forma integrada con el resto de los componentes del entrenamiento (Olmedilla y Domínguez-Igual, 2016). En este sentido son necesarios más estudios basados en intervenciones psicológicas que aporten una visión global del deportista, tanto en equipos como de casos individuales (Barker et al., 2013; Page y Thelwell, 2013).

En resumen, las transformaciones que ha sufrido el club en cuestiones de metodología y valores han obligado paralelamente a una transformación y adaptación cultural en materia de trabajo psicológico que ha requerido de varios años de constancia en la labor dentro de un club de fútbol, tal y como ha sucedido en otras ocasiones (García-Naveira y Jerez, 2012; Morilla et al., 2003), donde los aciertos y errores han sido y son claves del aprendizaje y crecimiento del trabajo psicológico en el deporte.

En este sentido, se hace necesario destacar la importancia que tras estos años de adaptación del rol del psicólogo en las categorías inferiores del Real Murcia C.F., se nos ha ido dotando al Área de Psicología de los recursos y medios disponibles, valorando en todo momento la necesidad que, para la Psicología del Deporte, tiene el trabajo interdisciplinar con el resto de profesionales que están en contacto continuo con el deportista y con el club y que, a la postre, enriquecen y refuerzan nuestra labor.

Si atendemos a las dificultades encontradas, la principal fue la de incluir y adaptar el Área de Psicología dentro de la estructura de funcionamiento, ya arraigada, en un club con más de 100 años de historia de trabajo. En este sentido, la dificultad radicó en implementar esta área y sus servicios no como una entidad independiente, supeditada a los demás servicios que proveía el club, sino dentro de una estructura de trabajo igualitaria e interdisciplinar, en la que hubiera retroalimentación continúa. Para este propósito fue necesaria la comunicación directa y habitual con los directivos del club y demás colaboradores, así como la inclusión del entrenamiento psicológico dentro del área de metodología del club.

Para finalizar y con el propósito de que este trabajo sirva de guía y ayuda a futuros psicólogos que quieran trabajar en el mundo del deporte, hemos de eliminar la imagen del psicólogo con bata y tras un ordenador. Es necesario estar presente en los campos de entrenamiento y ser "psicólogos de chándal". Hay que adaptar el trabajo a las circunstancias y facilitar que los deportistas y cuerpo técnico nos vean como un miembro más del cuerpo técnico, disponibles para ayudarlos ante cualquier dificultad, dentro y fuera del campo.

\section{Referencias}

Abenza, L., González, J., Reyes, L., Reyes, F., Blas, A. y Olmedilla, A. (2014). Descripción y evaluación del entrenamiento psicológico de una deportista de regata clase laser radial. Revista Iberoamericana de Psicología del Ejercicio y el Deporte, 9, 67-92.

Andreu, M. D. (2003). Programa de entrenamiento psicológico y metodología de trabajo en un equipo de fútbol juvenil. Cuadernos de Psicología del Deporte, 3, 83-94.

Bakeman, R. y Gottman, J.M. (1989). Observación de la interacción: Una introducción al análisis secuencial. Madrid, España: Morata.

Barker, J. B., Mellalieu, S. D., McCarthy, P. J., Jones, M. V. y Moran, A. (2013). A Review of Single-Case Research in Sport Psychology 1997-2012: Research Trends and Future Directions. Journal of Applied Sport Psychology, 25, 4-32. https://doi.org/10.108 0/10413200.2012.709579

Beck, A. T. (1976). Cognitive Therapy and the Emotional Disorders. New York, Estados Unidos: New American Library.

Blasco, T. (1994). Actividad física y salud. Barcelona, España: Martínez Roca.

Boixadós, M. y Valiente, L. (2007). Papel de los agentes de socialización en deportistas en edad escolar. Revista de Psicología del Deporte, 7, 295-310.

Bossio, M. R., Raimundi, M. J. y Correa, L. G. (2012). Programa de Entrenamiento en Habilidades Psicolgógicas en jugadoras de voleibol de alto rendimiento. Cuadernos de Psicología del Deporte, 12, 9-16.

Buceta, J. M. (1992). Intervención psicológica con el equipo nacional olímpico de baloncesto femenino. Revista de Psicología del Deporte, 7, 70-87.

Buceta, J. M. (2007). Intervención psicológica en el entrenamiento deportivo: estrategias para optimizar el funcionamiento de jugadores de baloncesto en sesiones de ensayo repetitivo. Revista de Psicología del Deporte, 8, 39-52.

Buceta, J. M. (2015). Mi hijo es el mejor, y además es mi hijo. Madrid, España: Dykinson.

Chicau, C., Silva, C. y Palmi, J. (2012). Programa de intervención psicológica para la optimización del concepto de equipo (team building) en jóvenes futbolistas. Revista de Psicología del Deporte, $21,49-58$. 
Cruz, J. (1992). El asesoramiento y la intervención psicológica en deportistas olímpicos. Revista de Psicología del Deporte, 1, 41-46.

Cruz, J. (1994). Asesoramiento psicológico a entrenadores: experiencia en baloncesto de iniciación. Apunts. Educació Física i Esports, 35, 5-14.

Cruz, J. (2001). Factores motivacionales en el deporte infantil y asesoramiento psicológico a entrenadores y padres. En. J. Cruz (Ed.), Psicología del Deporte (pp. 245-268). Madrid, España: Síntesis.

Cruz, J., Figueroa, J., Garcia-Mas, A. yTorregrosa, M. (2003). Design and application of a psychological campaign focusing on parents' positive participation in Sport. En R. Stelter (Ed.), Proceedings of the XIth European Congress of Sport Psychology. New approaches to Exercise and Sport Psychology: Theories, methods and applications (pp. 46-47). Denmark: Institute of Exercise and Sport Sciences.

Dosil, J. (2004). Psicología de la Actividad Física y del Deporte. Madrid, España: McGraw-Hill.

García-Naveira, A. (2010). El psicólogo del deporte en el alto rendimiento: aportaciones y retos futuros. Papeles del Psicólogo, 37, 259-268.

García-Naveira, A. (2017). Entrenamiento psicológico para la mejora del autocontrol en un entrenador de fútbol. Acción Psicológica, 14(1), 27-42. https://doi.org/10.5944/ap.14.1.19261

García-Naveira, A. y Jerez, P. (2012). Departamento de psicología del club Atlético de Madrid: filosofía, programación y desempeño profesional en el fútbol base. Cuadernos de Psicología del Deporte, 12(1), 111-120.

Gimeno, F. (2007). Descripción y evaluación preliminar de un programa de habilidades sociales y de solución de problemas con padres y entrenadores en el deporte infantil y juvenil. Revista de Psicología del Deporte, 12, 67-79.

Gimeno, F., Buceta, J. M. y Pérez-Llantada, M. (2001). El cuestionario Características Psicológicas Relacionadas con el Rendimiento Deportivo (CPRD): Características psicométricas. Análise Psicológica, 1(XIX), 93-133. https:// doi.org/10.14417/ap.346

Godoy-Izquierdo, D., Vélez, M. y Pradas, F. (2009). Nivel de dominio de las habilidades psicológicas en jóvenes jugadores de tenis de mesa, bádminton y fútbol. Revista de Psicología del Deporte, 18, 45-59.

Groves, D., Heekin, R. y Banks, C. (1978). Content analysis: International Journal of Sport Psychology. International Journal of Sport Psychology, 9, 1-6.

Hernández, J. M. (2005). Fundamentos del deporte: Análisis de las estructuras del juego deportivo. Zaragoza, España: Inde Publicaciones.

Jaenes, J. C., Caracuel, J. C. y Penaloza, R. (2012). Intervención en psicología del deporte: un caso de remo de alta competición. Revista de Psicología del Deporte, 27, 59-64.

Jordet, G. (2005). Perceptual training in soccer: An imagery intervention study with elite players. Journal of Applied Sport Psychology, 17, 140-156. https://doi.org/10.1080/10413200590932452

Junichi, M. y Hajime, K. (2007). Psychological skill training for the Japanese soccer team in 2005 Universiade game in Izmir. Journal of Sport Science and Medicine, 6(suppl. 10), 88

Lameiras, J., Almeida, P., Pons, J. y García-Mas, A. (2014). Incorporación de una rutina para la optimización del rendimiento deportivo. Revista de Psicología del Deporte, 23, 337-343.
Latinjak, A. T., Font-Lladó, R., Zourbanos, N. y Hatzigeorgiadis, A. (2016). Goal-Directed Self-Talk Interventions: A Single-Case Study With an Elite Athlete. The Sport Psychologist, 30, 189-194. https://doi.org/10.1123/tsp.2015-0120

Lorenzo, J. (2001). Manual de prácticas de psicología deportiva. Madrid, España: Biblioteca Nueva.

Lorenzo, J., Gómez, M. A., Pujals, C. y Lorenzo, A. (2012). Análisis de los efectos de un programa de intervención psicológica en jóvenes jugadores de baloncesto. Revista de Psicología del Deporte, 21, 43-48.

McArdle, S. y Moore, P. (2012). Applying evidence-based principles from CBT to sport psychology. The Sport Psychologist, 26, 299310. https://doi.org/10.1123/tsp.26.2.299

Medina, J. y Delgado, M. A. (1999). Metodología de entrenamiento de observadores para investigaciones sobre E.F. y deporte en las que se utilice como método la observación. Revista Motricidad, 5, 69-86.

Morilla, M., Pérez, E., Gamito, J. M., Gómez, M. A., Sánchez, J. E. y Valiente, M. (2001). Desarrollo de un programa de preparación psicológica con los equipos de un club de fútbol de la primera división española. Revista digital efdeportes, 7, 42. Recuperado de http://www.efdeportes.com/efd42/preps.htm

Morilla, M., Pérez, E., Gamito, J. M., Gómez, M. A., Sánchez, J. E. y Valiente, M. (2003). Planificación psicológica de la cantera del Sevilla F.C. S.A.D.: Organización, funcionamiento y programa deportivo-formativo. Cuadernos de Psicología del Deporte, 3(2), 17-30.

Munroe-Chandler, K. J., Murphy, L., Hall, C. R. y Fishburne, G. (Septiembre, 2007). Speed/accuracy trade-off: An explanation for soccer imagery intervention? Poster presentado en el 12th European Congress of Sport Psychology de la European Fedration of Sport Psychology. Halkidiki, Greece.

Olivares, J., Xavier, F. y Lozano, M. (2010). Técnicas para la adquisición de habilidades de enfrentamiento. En J. Olivares y F. Xavier (Eds.), Técnicas de modificación de conducta (pp. 443-481). Madrid, España: Biblioteca Nueva.

Olmedilla, A., Ortega, E., Ortín, F. J. y Andreu, M. D. (2008). Entrenamiento psicológico en fútbol base de élite: percepción de aplicabilidad e índices de satisfacción. Revista Iberoamericana de Psicología del Ejercicio y el Deporte, 3(1), 35-52.

Olmedilla, A., Bazaco, M. J., Ortega, E. y Boladeras, A. (2011). Formación psicológica en futbolistas juveniles: relación con el bienestar psicológico percibido, la ansiedad y la satisfacción. Revista Científica Electrónica de Psicología, 12, 221-237.

Olmedilla, A., Ortega, E., Andreu, M. D. y Ortín, F. J. (2010). Programa de intervención psicológica en futbolistas: evaluación de habilidades psicológicas mediante el CPRD. Revista de Psicología del Deporte, 19, 249-262.

Olmedilla, A., Ortega, E., Boladeras, A., Ortín, F. J., y Bazaco, M. J. (2013). Entrenamiento en estrategias y técnicas psicológicas y percepción de ayuda en futbolistas juveniles. SPORT TK-Revista Euroamericana de Ciencias del Deporte, 2(1), 51-58.

Olmedilla, A. y Domiguez-Igual, J. (2016). Entrenamiento psicológico para la mejora de la atención y autoconfianza en un futbolista. Revista de Psicología Aplicada al Deporte y al Ejercicio Físico, 1, e4. http://dx.doi.org/10.5093/rpadef2016a4

Ortín, F. J., Maestre, M. y García-de-Alcaraz, A. (2016). Formación a entrenadores de fútbol base y grado de satisfacción de los 
deportistas. SPORT TK-Revista EuroAmericana de Ciencias del Deporte, 5(1), 11-17.

Page, J. y Thelwell, R. (2013). The value of social validation in single-case methods in Sport and Exercise Psychology. Journal of Applied Sport Psychology, 25, 61-71. https://doi.org/10.1080/10 413200.2012 .663859

Peris, D. (marzo, 2008). Estrategias psicológicas para la intervención con padres y madres de deportistas. Taller aplicado presentado en el XI Congreso Nacional y Andaluz y III Congreso Iberoamericano de Psicología de la Actividad Física y del Deporte de la Universidad Pablo Olavide, Sevilla.

Pujals, C. y Jodra, P. (2011). Evolución de los aspectos psicológicos en jugadores de baloncesto infantil durante una Temporada de competición. Revista Iberoamericana de Psicología del Ejercicio y el Deporte, 6(1), 23-30.

Ramirez, J. y Diaz, M. (2004). El papel educativo y la responsabilidad de los entrenadores deportivos. Una investigación sobre la instrucciones a escolares en fútbol de competición. Revista de Educación, 335, 163-187.

Romero, A. E., Zapata, R., García-Mas, A., Brustad, R. J., Garrido, R. y Letelier, A. (2010). Estrategias de afrontamiento y bienestar psicológico en jóvenes tenistas de competición. Revista de Psicología del Deporte, 19(1), 117-133.

Salgado, R., Rivas, C.A. y García-Mas, A. (2011). Aparición del burnout en jugadores de fútbol de primera división profesional en México: estrategias de intervención. Cuadernos de Psicología del Deporte, 17(2, Supl.), 57-62.

Sheard, M. y Golby, J. (2006). Effect of a psychological skills training program on swimming performance and positive psychological development. International Journal of Sport and Exercise Psychology, 4, 149-169. https://doi.org/10.1080/1612197X.2006.9671790

Sousa, C., Cruz, J., Torregrosa, M., Vilches, D. y Viladrich, C. (2006). Evaluación conductual y programa de asesoramiento personalizado a entrenadores (PAPE) de deportistas jóvenes. Revista de Psicología del Deporte, 15, 263-278.
Sousa, C., Cruz, J., Viladrich, C. y Torregrosa, M. (2007). Efectos del programa de asesoramiento personalizado a entrenadores (PAPE) en el compromiso deportivo y el abandono de futbolistas jóvenes. Motricidad. European Journal of Human Movement, 19.

Spielberger, C. (1973). Manual for State-Trait Anxiety Inventory for Children. Palo Alto, CA, Estados Unidos: Consulting Psychologists Press.

Torregrosa, M. y Cruz, J. (2006). El deporte de iniciación como base de la carrera deportiva de adultos activos y deportistas de élite. En E.J. Garcés, A. Olmedilla y P. Jara (Eds.), Psicología y Deporte (pp. 585-602). Murcia, España: Diego Marín.

Turner, M. J., Slater, M. J. y Barker, J. B. (2014). Not the end of the world: The effects of rational-emotive behavior therapy (REBT) on irrational beliefs in elite soccer academy athletes. Journal of Applied Sport Psychology, 26, 144-156. https://doi.org/10.1080/ 10413200.2013 .812159

Ureña, P. (2003). El entrenamiento psicológico en el deporte de alto rendimiento. Revista ABRA, 23(32), 85-93.

Valdés, H. M. (1996). La preparación Psicológica del Deportista. Barcelona, España: Inde Publicaciones.

Vives, L. y Garcés, E. J. (2003). Intervención psicológica en un club de fútbol base: propuesta de un sistema de actuación psicológica desde sus diversas áreas. Cuadernos de Psicología del Deporte, 3(2), 51-66.

Weinberg, R.S. y Gould, D. (2010). Fundamentos de Psicología del Deporte y del Ejercicio físico. Madrid, España: Ed. Médica Panamericana.

Wood, G. y Wilson, M.R. (2012). Quiet-eye training, perceived control and performing under pressure. Psychology of Sport and Exercise, 13, 721-728. https://doi.org/10.1016/j.psychsport.2012.05.003

Wright, D. J., McCormick, S. A., Birks, S., Loporto, M. y Holmes, P. S. (2015). Action observation and imagery training improve the ease with which athletes can generate imagery. Journal of Applied Sport Psychology, 27, 156-170. https://doi.org/10.1080/ 10413200.2014.968294 